\title{
Fatores associados ao desenvolvimento da anomalia congênita em recém-nascidos
}

\author{
Factors associated with the development of congenital anomalies in newborns \\ Factores asociados al desarrollo de anomalía congénita en recién nacidos
}

Maria Eduarda Wanderley de Barros Silva ORCID: https://orcid.org/0000-0002-4642-3282 Universidade Federal de Campina Grande, Brasil

E-mail: Eduarda.wanderley@outlook.com

Maria Letícia Cardoso da Silva Barbosa

ORCID: https://orcid.org/0000-0002-2935-6882

Universidade Federal de Campina Grande, Brasil

E-mail: marialeticia20151@hotmail.com

Thaís Andrade dos Santos

ORCID: https://orcid.org/0000-0002-9669-7468

Universidade Federal de Pernambuco, Brasil E-mail: thais.andradesantos@ufpe.br

Daniela de Lira Silva

ORCID: https://orcid.org/0000-0001-8145-2508

Universidade Federal de Pernambuco, Brasil E-mail: daniela.lirasilva@ufpe.com.br

Vitória Maria de Arruda Passos

ORCID: https://orcid.org/0000-0003-4241-3762

Universidade Federal de Pernambuco, Brasil

E-mail: vitoriaaapassos@gmail.com

Clara Gomes Carvalho Silva

ORCID: https://orcid.org/0000-0001-6039-525X

Universidade Federal de Pernambuco, Brasil

E-mail: claragomescarvalhosud@gmail.com

Natália Valença Cavalcante

ORCID: https://orcid.org/0000-0003-0428-9529

Universidade Federal de Pernambuco, Brasil

E-mail: natalia.valencac@ufpe.br

Jennifer Martins Pereira

ORCID: https://orcid.org/0000-0001-9305-9877

Universidade Estadual de Maringá, Brasil

E-mail: jennifermartins25pereira@gmail.com

Ana Paula Moura Manzin

ORCID: https://orcid.org/0000-0003-1864-7024

Universidade Tiradentes, Brasil

E-mail: apmmanzini@gmail.com

Gabriel Fragoso Peixoto

ORCID: https://orcid.org/0000-0003-0640-6602

Universidade Tiradentes, Brasil

E-mail: gabriel.fpeixoto@souunit.com.br

Christian Cleber Maux Calheiros

ORCID: https://orcid.org/0000-0002-0125-3451

Universidade Tiradentes, Brasil

E-mail: christiancalheiros@gmail.com

Jones Pinto da Silva Neto

ORCID: https://orcid.org/0000-0001-9449-1960 Escola de Ensino Superior do Agreste Paraibano, Brasil E-mail: jonesneto.pb@gmail.com

Lindinês Pereira de Macêdo

ORCID: https://orcid.org/0000-0001-8421-6527

Universidade Federal de Campina Grande, Brasil E-mail: lindinezpereira09gmail.com

Angela Ferreira da Silva

ORCID: https://orcid.org/0000-0001-8279-7559

Universidade Federal de Pernambuco, Brasil

E-mail: angela96ferreira@hotmail.com

Larissa Lima Soares

ORCID: https://orcid.org/0000-0002-7682-372X

Universidade Tiradentes, Brasil

E-mail: larissalyma@hotmail.com 


\title{
Resumo
}

As falhas congênitas são condições patológicas que são determinadas por fatores que surgem concomitante ao nascimento, durante ou depois da concepção. No entanto, as anomalias também podem ser desenvolvidas por diversos fatores, idade materna, fatores ambientais, alterações do desenvolvimento dos tecidos e também por agentes infecciosos. As alterações congênitas podem estar associadas por uma variedade de fatores etiológicos. $\mathrm{O}$ estudo visa apresentar resultados encontrados na revisão integrativa qualitativa, que apresentem os fatores associados anomalias congênitas, expondo as diferentes vias as quais essas manifestações podem se desenvolver. A pesquisa trata-se de uma revisão integrativa da literatura do tipo qualitativa, a elaboração de levantamento metodológico para a pesquisa foi realizada no período Novembro de 2021, a base de dados utilizada foi Medical Literature Analysis and Retrieval System Online (MEDLINE) e Scientific Electronic Library Online (SCIELO). Utilizando o Medical Subject Headings (MeSH) e os Descritores de ciências em saúde (DeCS), são evidentes que há diversos fatores os quais podem causar o desenvolvimento de anomalias congênitas, tendo em vista os resultados, o cuidado e atenção ao período do pré-natal é fundamental, para realização de diagnósticos e intervenções diante as anomalias, no entanto, quando a existente após nascimento as principais medidas para que se tenha um cuidado neuroprotetor neonatal é necessário a proteção do sono, parceira com as famílias e um ambiente terapêutico a fim de minimizar o estresse e dor. É necessário que seja disseminado o conhecimento sobre as anomalias e suas causas para que assim muitos causos sejam evitados, apesar da etiologia genética ser um fator contribuinte por uma porcentagem considerável nas anomalias congênitas deve-se levar em consideração os fatores evitáveis como as infecções que podem ser transmitidas ao feto e endocrinopatias maternas. Palavras-chave: Anomalia congênita; Neonatologia; Cuidados de enfermagem.

\begin{abstract}
Congenital defects are pathological conditions that are determined by factors that arise concurrently with birth, during or after conception. However, anomalies can also be developed by several factors, maternal age, environmental factors, alterations in tissue development and also by infectious agents. Congenital alterations can be associated by a variety of etiological factors. The study aims to present results found in the qualitative integrative review, which present the factors associated with congenital anomalies, exposing the different ways in which these manifestations can develop. The research is an integrative literature review of the qualitative type, the preparation of a methodological survey for the research was carried out from November 4, 2021 to November 9, 2021, the database used was Medical Literature Analysis and Retrieval System Online (MEDLINE) and Scientific Electronic Library Online (SCIELO). Using the Medical Subject Headings (MeSH) and the Health Sciences Descriptors (DeCS). it is clear that there are several factors which can cause the development of congenital anomalies, considering the results, care and attention to the prenatal period is essential to carry out diagnoses and interventions in the face of anomalies, however, when will Existing after birth, the main measures for having a neonatal neuroprotective care are sleep protection, partnership with families and a therapeutic environment in order to minimize stress and pain. It is necessary to disseminate knowledge about the anomalies and their causes so that many causes are avoided, despite the genetic etiology being a contributing factor for a considerable percentage of congenital anomalies, avoidable factors such as infections that can be taken into account be transmitted to the fetus and maternal endocrinopathies.
\end{abstract}

Keywords: Congenital anomaly; Neonatology; Nursing care.

\section{Resumen}

Como los defectos congénitos son condiciones patológicas que están determinadas por factores que surgen concurrentemente con el nacimiento o durante nuestra concepción. Sin embargo, como las anomalías también pueden ser desarrolladas por diversos factores, la vida materna, factores ambientales, alteraciones en el desarrollo de dos tejidos y también por agentes infecciosos. Los cambios congénitos pueden estar asociados con una variedad de factores etiológicos. En otras palabras, este estudio tiene como objetivo presentar los resultados encontrados en una revisión cualitativa integradora, que revela los factores asociados a las anomalías congénitas, exponiendo las diferentes formas en que estas manifestaciones pueden desarrollarse. La investigación es una revisión integradora de la literatura del tipo cualitativo, la elaboración de la encuesta metodológica para la investigación se realizó del 4 de noviembre de 2021 al 9 de noviembre de 2021, con base en los datos utilizados para el Análisis y Sistema. Recuperación de Literatura Médica en Línea (MEDLINE) y Scientific Electronic Library Online (SCIELO). Uso de títulos de materias médicas (MeSH) y descriptores de ciencias de la salud (DeCS). Es evidente que existen varios factores que pueden ocasionar o desarrollar anomalías congénitas, a la vista de los resultados, es decir, el cuidado y atención a la atención prenatal e imprescindible para la realización de diagnósticos e intervenciones contra las anomalías, sin embargo, cuando el Temprano El nacimiento como las principales medidas para tener un cuidado neuroprotector neonatal es necesario para proteger el sueño, asociarse con las familias y un ambiente terapéutico con el fin de minimizar el estrés y el sueño. Es necesario que se difunda el conocimiento sobre las anomalías y sus causas para que se eviten muchas causas, a pesar de la etiología genética, será un factor que contribuya en un porcentaje considerado a anomalías congénitas que deben ser tomadas en consideración por los padres. Evitas infecciones que podemos transmitir al feto y endocrinopatías maternas.

Palabras clave: Anomalía congenital; Neonatología; Cuidado de enfermera. 


\section{Introdução}

As falhas congênitas são condições patológicas que são determinadas por fatores que surgem concomitante ao nascimento, durante ou depois da concepção. O significado da palavra congênita expressa que há um defeito básico, mas, não necessariamente identificado no nascimento, ou seja, pode vir a serem más formações internas ou funcionais, que podem se apresentar ao decorrer do desenvolvimento humano (Alberto et al., 2010).

O desenvolvimento do embrião é facilmente perturbado por ser muito sensível durante a formação dos tecidos e órgãos, as anomalias se apresentam nesse processo e para determinar o tipo de má formação, vale considerar o tipo de tecido o qual foi perturbado durante sua evolução. Nos humanos, o sistema nervoso aparece juntamente com o coração, uma sensibilidade mais prematura, com maior vulnerabilidade para doenças do tubo neural que acontecem durante o período de 15 a 30 dias, visto que o fechamento do tubo neural ocorre entre o $23^{\circ}$ e $26^{\circ}$ para o neuroporo cefálico e $26^{\circ}$ e $30^{\circ}$ para o neuroporo caudal. Os erros no processo de fechamento do tudo neural atrapalham a diferenciação do sistema nervoso central e deste modo, acarretar diversas anomalias humanas (Alberto et al., 2010). Propõe-se que diversos genes estejam envolvidos no fechamento do tubo neural. E alguns desses genes podem ser um forte componente genético, enquanto os demais podem gerar apenas um pequeno efeito ou interagir com outros genes (Aguiar et al., 2003).

No entanto, as anomalias também podem ser desenvolvidas por diversos fatores, idade materna, fatores ambientais, alterações do desenvolvimento dos tecidos e também por agentes infecciosos. Apesar de o concepto estar altamente protegido pelas membranas que envolvem o feto, os agentes infecciosos maternos podem eventualmente atingi-los, dessa forma, os efeitos desses agentes possuem efeitos divergentes dos ambientais, por serem muitos persistentes e suas manifestações podem se tornar aparente somente após o nascimento (FIOCRUZ, 1981).

As alterações congênitas podem estar associadas por uma variedade de fatores etiológicos. As anomalias mais presentes na população segundo o Ministério da Saúde são oriundas de: defeitos do tubo neural, microcefalia, fendas orais, cardiopatias congênitas, anomalias de órgãos genitais, defeitos de membros, síndrome de Down e defeitos da parede abdominal. No entanto, muitas das anormalidades podem ser prevenidas, por meio de estratégias como controle de doenças maternas, vacinação e exposições a fatores de riscos, como tabagismo, alcoolismo e substâncias ilícitas. Quando detectadas precocemente algumas possuem tratamento existente e intervenções efetivas (Ministério da Saúde, 2021).

A pobreza relacionada aos defeitos congênitos é bastante variada, inespecífico, indireto e inclui condições ambientais como: violência, estresse, poluição, falta de exames pré-natais, comportamentos reprodutivos adversos, ausência de informações sobre a prevenção durante a gravidez, condições de vida, como exposição ao tabaco e álcool, e a falta de acesso aos serviços de saúde no período gestacional (Pawluk et al., 2014).

Os tipos de anomalias congênitas são considerados como leve, moderados e graves. As anomalias moderadas a graves interferem na qualidade de vida da criança, além de ocasionarem traumas familiares e dificuldade de adaptação à sociedade (Laurenti et al., 2014).

As malformações maiores e múltiplas são mais predominante na população branca, outros fatores e risco também que foram destacados na literatura científica abordam: a idade materna avançada (>35 anos), maior número de gestações anteriores, uso indevido de fármacos, parto cesáreo, menor duração da gestação, tabagismo, ingestão de bebida alcoólica, deficiências nutricionais, presença de doenças preexistentes, exposição ocupacional a agentes teratogênicos, condições socioeconômicas desfavoráveis, baixo peso ao nascer e menor escore de Apgar (Andrade et al., 2017).

O impacto das anomalias congênitas referente a mortalidade infantil depende de diversos fatores, que incluem a prevalência das anomalias, a qualidade e a disponibilidade de tratamento médico e cirúrgico, também incluem a eficácia das medidas de prevenção na atenção primária, acompanhamento e vacinação da gestante, durante o período do pré-natal. Esse 
acompanhamento qualificado permite a investigação do desenvolvimento morfológico fetal, doenças na gestação e do feto possam ser identificadas em fase precoce (Arruda et al., 2008).

No período do pré-natal é de extrema importância que seja identificado diagnóstico de anomalias digestivas e da parede abdominal, pois, na maioria dos casos é possível ainda a interrupção da gravidez caso desejado ou se manter adiante até o nascimento, encaminhar para um centro com cuidados perinatais diferenciados. A presença de uma má formação congênita, embora seja rara que se apresente individualmente, no seu conjunto se torna mais recorrente e exige uma equipe multiprofissional de saúde (Rocha et al., 2004).

As intervenções de promoção de saúde, só se tornam viáveis com o conhecimento profissional sobre os fatores que estão relacionados ao desenvolvimento de anomalias congênitas. A análise situacional na gestação e esse conhecimento se tornam uma ferramenta essencial para a proteção e a realização de condutas no período pré-concepcional e gestacional, assim como ações durante o período do parto. O Brasil, assim como outros países, criou programas e ações preventivas de malformações congênitas. Deste modo, desde 2002 acontece a fortificação de farinhas de trigo e milho com ácido fólico, também conta com a concretização de programas que preconizam o uso do ácido fólico de modo adequado durante o período gestacional, com o intuito de diminuir os casos de falhas no tubo neural do feto (Andrade et al., 2017).

Deste modo, considerando todas as informações sobre o tema, o estudo visa apresentar resultados encontrados na revisão integrativa qualitativa, que apresentem os fatores associados anomalias congênitas, expondo as diferentes vias as quais essas manifestações podem se desenvolver, desde os fatores externos/ambientais aos processos internos durante o período gestacional. Sendo assim, esse trabalho tem a relevância de esclarecer os fatores associados a anomalias congênitas, com o intuito de auxiliar a diagnósticos e investigações precoces e preventivas, fortalecendo assim, a assistência primária durante o início do pré-natal até o convívio e tratamento das patologias.

\section{Metodologia}

A pesquisa trata-se de uma revisão integrativa da literatura do tipo qualitativa, a qual a classificação permite uma amplitude de abordagem metodológica pertencente às revisões, permitindo a inclusão de estudos experimentais e nãoexperimentais para uma compreensão completa do fenômeno analisado. Sendo assim, foram realizadas as etapas 1- Elaboração da pergunta norteadora; 2- Amostragem da literatura; 3- Coleta de dados; 4- Análise crítica dos estudos incluídos; 5- Discussão dos resultados; 6-Apresentação da revisão/conclusão (Souza et al., 2010). Sendo assim, foi possível estruturar a pergunta norteadora: "Quais os fatores associados ao desenvolvimento da anomalia congênita em recém-nascido?".

A elaboração de levantamento metodológico para a pesquisa foi realizada no período de Novembro, as bases de dados utilizadas foram: Medical Literature Analysis and Retrieval System Online (MEDLINE) e Scientific Electronic Library Online (SCIELO). Utilizando o Medical Subject Headings (MeSH) e os Descritores de ciências em saúde (DeCS). Os descritores foram: Abnormalities Congenital, Neonatology e Nursingcare. Utilizando o cruzamento na base de dados com o operador booleano “AND”, Abnormalities Congenital AND Neonatology AND Nursingcare. Considerando os subtítulos anormalidade, enfermagem, fisiopatologia e prevenção e controle. Foram selecionados estudos do tipo revisão e análise, publicados nos últimos cinco anos e disponíveis gratuitamente na íntegra, após a filtração foram apresentados 49 estudos identificados no MEDLINE e 10 disponibilizados na plataforma SciELO, os quais passaram pela análise dos resumos e critérios de elegibilidade.

Em seguida, a partir da leitura dos resumos foi selecionado um quantitativo de estudos relacionados à pergunta norteadora, sendo encontrados artigos no idioma português e inglês, mas, com predominância da literatura inglesa disponibilizado nas bases de dados e posteriormente traduzida para o português e apresentados o quantitativo e processo da seleção na Figura 1 a seguir: 
Figura 1 - Fluxograma de seleção dos estudos, segundo o Preferred ReportingItems for Systematic Reviews and Meta Analyses (PRISMA). Natal, Rio Grande do Norte, Brasil, 2021.

\section{PERCURSO DE SELEÇÃO DOS ESTUDOS}

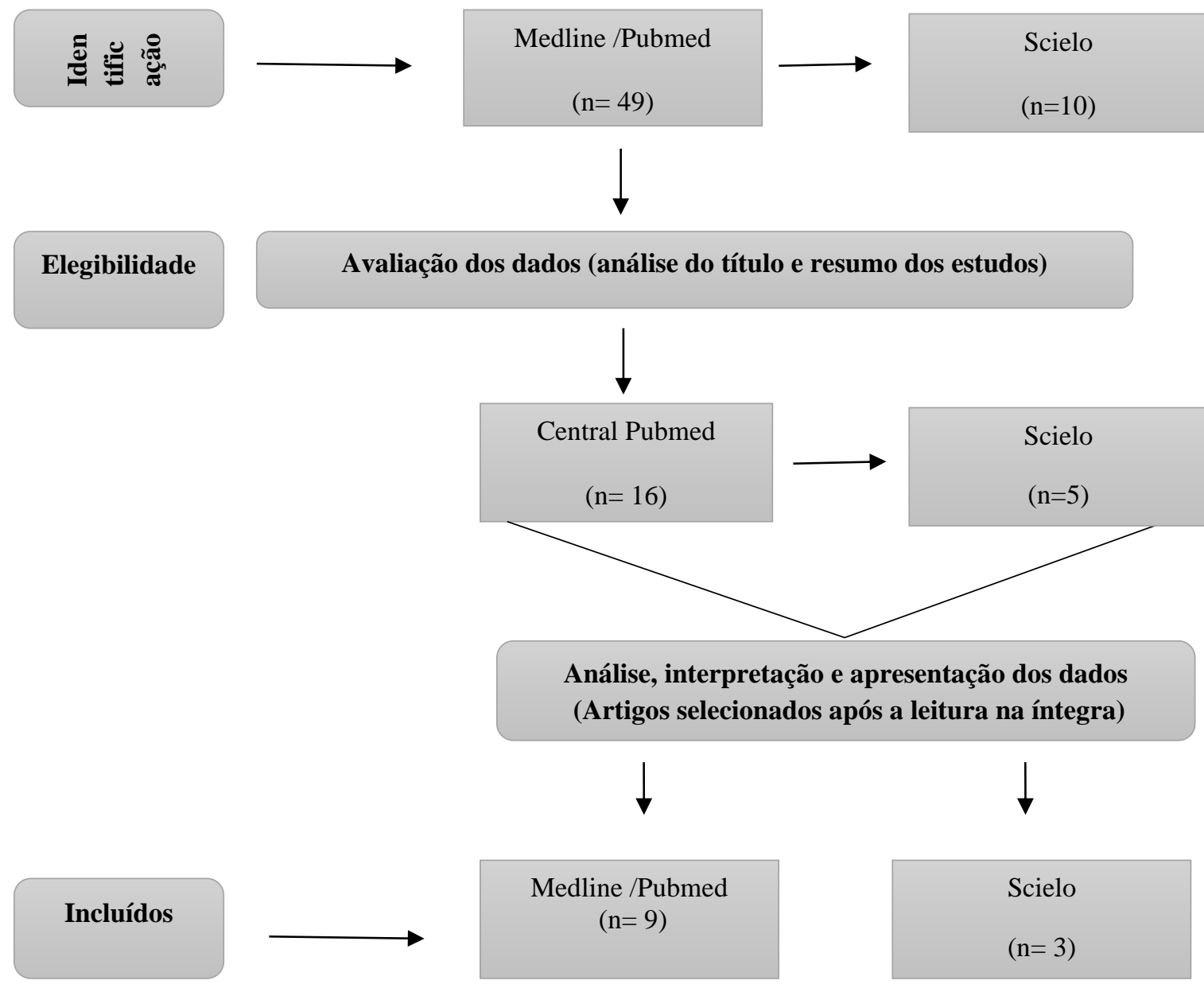

Fonte: Autores.

\section{Resultados e Discussão}

Os estudos mais atuais foram agrupados no Quadro 1, contendo informações sobre autoria, ano da publicação, título e resultados. Objetivando assim, uma coleta de estudos atualizados e proporcionando uma discussão na revisão integrativa referente aos fatores associados ao desenvolvimento da anomalia congênita em recém-nascidos. Sendo assim, foi elaborado o corpus de análise da pesquisa representados no quadro abaixo: 
Quadro 1. Corpus de análise de pesquisa. Brasil, 2021.

\begin{tabular}{|c|c|c|c|c|}
\hline $\begin{array}{l}\text { Autoria e ano } \\
\text { de publicação }\end{array}$ & Título do artigo & Objetivo do estudo & $\begin{array}{c}\text { Delineamento } \\
\text { metodológico }\end{array}$ & Resultados \\
\hline $\begin{array}{l}\text { ATLAW, D. et } \\
\text { al., } 2021 .\end{array}$ & $\begin{array}{c}\text { Magnitude and } \\
\text { determinants of neural } \\
\text { tube defect in Africa: a } \\
\text { systematic review and } \\
\text { meta-analysis }\end{array}$ & $\begin{array}{l}\text { Determinar a prevalência e os } \\
\text { fatores associados aos defeitos } \\
\text { do tubo neural na África. }\end{array}$ & $\begin{array}{c}\text { Revisão } \\
\text { sistemática. }\end{array}$ & $\begin{array}{l}\text { A prevalência combinada dos defeitos do tubo neural } \\
\text { na África foi considerada alta. A exposição materna a } \\
\text { pesticidas e radiação de raios-X foram } \\
\text { significativamente associadas com os defeitos do tubo } \\
\text { neural. A suplementação de ácido fólico antes e dentro } \\
\text { do primeiro mês de gravidez foi considerada um fator } \\
\text { de proteção. }\end{array}$ \\
\hline $\begin{array}{l}\text { AVSAR, T. S. } \\
\text { et al., } 2021 .\end{array}$ & $\begin{array}{l}\text { Health outcomes of } \\
\text { smoking during } \\
\text { pregnancy and the } \\
\text { postpartum period: an } \\
\text { umbrella review }\end{array}$ & $\begin{array}{l}\text { Identificar as consequências de } \\
\text { fumar durante a gravidez e no } \\
\text { período pós-parto tem } \\
\text { consequências graves para a } \\
\text { saúde da mãe e do bebê. }\end{array}$ & $\begin{array}{c}\text { Revisão } \\
\text { sistemática. }\end{array}$ & $\begin{array}{c}\text { Mostrou que fumar durante a gravidez e no período } \\
\text { pós-parto tem consequências significativas para a } \\
\text { saúde de mães e bebês. É importante encorajar as } \\
\text { grávidas fumantes a parar de fumar ou reduzir o } \\
\text { número de cigarros consumidos, caso não estejam } \\
\text { preparadas para parar totalmente, uma vez que as } \\
\text { evidências existentes indicam uma associação dose- } \\
\text { resposta. }\end{array}$ \\
\hline $\begin{array}{l}\text { WINDT, M. V. } \\
\text { D. et al., } 2021 \text {. }\end{array}$ & $\begin{array}{l}\text { Epidemiology and } \\
\text { (Patho) physiology of } \\
\text { folic acid supplement } \\
\text { use in obese women } \\
\text { before and during } \\
\text { pregnancy }\end{array}$ & $\begin{array}{l}\text { Identificar uma visão geral da } \\
\text { epidemiologia da deficiência de } \\
\text { folato em mulheres (pré) } \\
\text { grávidas obesas, elaborar sobre } \\
\text { os mecanismos potenciais } \\
\text { subjacentes à deficiência de } \\
\text { folato e discutir considerações } \\
\text { para o uso de doses mais altas } \\
\text { de suplementos de ácido fólico. } \\
\end{array}$ & $\begin{array}{l}\text { Estudo de } \\
\text { metanálise, } \\
\text { revisão } \\
\text { sistemática. }\end{array}$ & $\begin{array}{l}\text { A evidência científica do envolvimento de várias vias } \\
\text { relacionadas ao folato implica no aumento da } \\
\text { suplementação de ácido fólico recomendada em } \\
\text { mulheres obesas. Contudo, a absorção fisiológica de } \\
\text { ácido fólico sintético é limitada e os efeitos colaterais } \\
\text { do ácido fólico não metabolizado em mães e filhos, em } \\
\text { particular variações na reprogramação epigenética com } \\
\text { efeitos de longo prazo na saúde. }\end{array}$ \\
\hline $\begin{array}{l}\text { HELLE, E. et } \\
a l ., 2020 .\end{array}$ & $\begin{array}{l}\text { Maternal obesity and } \\
\text { diabetes mellitus as } \\
\text { risk factors for } \\
\text { congenital heart disease } \\
\text { in offspring }\end{array}$ & $\begin{array}{c}\text { Revisar as evidências } \\
\text { epidemiológicas e as } \\
\text { observações clínicas que } \\
\text { relacionam a obesidade materna } \\
\text { e o diabetes mellitus ao risco de } \\
\text { filhos com doença coronariana, } \\
\text { com atenção particular aos } \\
\text { modelos mecanísticos de } \\
\text { transmissão de risco materno- } \\
\text { fetal e distúrbios no primeiro } \\
\text { trimestre do desenvolvimento } \\
\text { cardíaco fetal. }\end{array}$ & $\begin{array}{l}\text { Estudo de } \\
\text { metanálise. }\end{array}$ & $\begin{array}{c}\text { Desnutrição materna e especialmente a deficiência de } \\
\text { folato têm sido associadas à doença cardíaca congênita } \\
\text { na prole, e há evidências de que mães obesas podem ter } \\
\text { uma resposta insuficiente à suplementação de ácido } \\
\text { fólico para prevenção primária de anomalias } \\
\text { congênitas. }\end{array}$ \\
\hline $\begin{array}{l}\text { OHLSSON, A. } \\
\text { et al., } 2020 .\end{array}$ & $\begin{array}{l}\text { Ibuprofeno para a } \\
\text { prevenção de } \\
\text { persistência do canal } \\
\text { arterial em bebês } \\
\text { prematuros e/ou com } \\
\text { baixo peso ao nascer }\end{array}$ & $\begin{array}{c}\text { Determinar a eficácia e } \\
\text { segurança do ibuprofeno em } \\
\text { comparação com placebo ou } \\
\text { outras drogas inibidoras da } \\
\text { ciclooxigenase na prevenção da } \\
\text { persistência do canal arterial em } \\
\text { bebês prematuros. }\end{array}$ & $\begin{array}{l}\text { Estudo de } \\
\text { metanálise. }\end{array}$ & $\begin{array}{l}\text { O uso profilático de ibuprofeno, em comparação com } \\
\text { placebo ou nenhuma intervenção, provavelmente } \\
\text { diminui a incidência de persistência do canal arterial, a } \\
\text { necessidade de tratamento de resgate com inibidores da } \\
\text { ciclooxigenase e de fechamento ductal cirúrgico. Os } \\
\text { efeitos adversos associados ao ibuprofeno (IV ou oral) } \\
\text { incluíram aumento do risco de oligúria, aumento dos } \\
\text { níveis de creatinina sérica e aumento do risco de } \\
\text { hemorragia gastrointestinal. }\end{array}$ \\
\hline $\begin{array}{l}\text { PETERSON, J. } \\
\text { K. et al., } 2017 .\end{array}$ & $\begin{array}{l}\text { Developmentally } \\
\text { supportive care in } \\
\text { congenital heart } \\
\text { disease: a concept } \\
\text { analysis }\end{array}$ & $\begin{array}{l}\text { Apresentar uma análise de } \\
\text { conceito usando o método de } \\
\text { Walker e Avant, a fim de } \\
\text { identificar e definir as } \\
\text { características dos cuidados de } \\
\text { suporte ao desenvolvimento, já } \\
\text { que podem ser aplicados à } \\
\text { população de neonatos, bebês e } \\
\text { crianças com cardiopatia } \\
\text { congênita. }\end{array}$ & $\begin{array}{l}\text { Análise de } \\
\text { conceito. }\end{array}$ & $\begin{array}{l}\text { De acordo com o que foi encontrado é necessário } \\
\text { implementar cuidados de alta qualidade centrados na } \\
\text { família que atendam às necessidades individuais de } \\
\text { desenvolvimento em uma população com alto risco de } \\
\text { sequelas de desenvolvimento. }\end{array}$ \\
\hline $\begin{array}{l}\text { LIMA, L. M. } \\
\text { M., VIANNA, } \\
\text { R. P. T. \& } \\
\text { MORAES, R. } \\
\text { M., 2019. } \\
\end{array}$ & $\begin{array}{l}\text { Identificação das } \\
\text { anomalias congênitas } \\
\text { baseado em um modelo } \\
\text { de decisão a partir de } \\
\text { redes neurais artificiais }\end{array}$ & $\begin{array}{l}\text { Identificar os tipos de } \\
\text { anomalias congênitas do } \\
\text { sistema nervoso a partir de um } \\
\text { modelo de tomada de decisão. }\end{array}$ & $\begin{array}{c}\text { Estudo } \\
\text { retrospectivo. }\end{array}$ & $\begin{array}{l}\text { O modelo utilizado para identificar as anomalias } \\
\text { congênitas do sistema nervoso pode ser útil na tomada } \\
\text { de decisão dos profissionais de saúde }\end{array}$ \\
\hline $\begin{array}{l}\text { COSME, H. } \\
\text { W., LIMA, L. } \\
\text { S. \& } \\
\text { BARBOSA, L. } \\
\text { G., } 2017 .\end{array}$ & $\begin{array}{l}\text { Prevalência de } \\
\text { anomalias congênitas e } \\
\text { fatores associados em } \\
\text { recém-nascidos do } \\
\text { município de São Paulo } \\
\text { no período de } 2010 \text { a } \\
\quad 2014 .\end{array}$ & $\begin{array}{l}\text { Estudar a prevalência de } \\
\text { anomalias congênitas em } \\
\text { nascidos em maternidades do } \\
\text { município de São Paulo, no } \\
\text { período de } 2010 \text { a } 2014 \text {. }\end{array}$ & $\begin{array}{c}\text { Estudo } \\
\text { transversal. }\end{array}$ & $\begin{array}{c}\text { As anomalias congênitas são causa de } \\
\text { morbimortalidade no período neonatal, e o seu } \\
\text { diagnóstico precoce é para o planejamento e a alocação } \\
\text { de recursos dos serviços de saúde especializados. }\end{array}$ \\
\hline
\end{tabular}




\begin{tabular}{|c|c|c|c|c|}
\hline $\begin{array}{l}\text { GONÇALVES, } \\
\text { M. K. S. et al., } \\
2021 .\end{array}$ & $\begin{array}{l}\text { Prevalência e fatores } \\
\text { associados as } \\
\text { malformações } \\
\text { congênitas em nascidos } \\
\text { vivos }\end{array}$ & $\begin{array}{c}\text { Estimar a prevalência de } \\
\text { malformações congênitas e } \\
\text { identificar os fatores associados } \\
\text { em nascidos vivos. }\end{array}$ & $\begin{array}{c}\text { Estudo } \\
\text { transversal. }\end{array}$ & $\begin{array}{l}\text { As variáveis apontadas no estudo integraram um } \\
\text { modelo preditivo que pode auxiliar no planejamento } \\
\text { dos serviços de saúde, sugerir hipóteses sobre os } \\
\text { fatores etiológicos, e subsidiar as ações do pré-natal } \\
\text { com atenção para os fatores identificados. }\end{array}$ \\
\hline $\begin{array}{l}\text { SANTOS, A. } \\
\text { C. C. \& } \\
\text { FRANÇA, G. } \\
\text { V. A., } 2021 .\end{array}$ & $\begin{array}{l}\text { Lista de anomalias } \\
\text { congênitas prioritárias } \\
\text { para a vigilância no } \\
\text { âmbito do Sistema de } \\
\text { informação sobre } \\
\text { nascidos vivos do } \\
\text { Brasil } \\
\end{array}$ & $\begin{array}{l}\text { Definir a lista de anomalias } \\
\text { congênitas prioritárias para o } \\
\text { aprimoramento do registro no } \\
\text { Sistema de Informações sobre } \\
\text { Nascidos Vivos (Sinasc). }\end{array}$ & $\begin{array}{c}\text { Estudo } \\
\text { documental. }\end{array}$ & $\begin{array}{c}\text { Dadas as complexidades envolvidas no diagnóstico e } \\
\text { notificação das anomalias com base no Sinasc, no } \\
\text { contexto de um país social e demograficamente } \\
\text { diverso, populoso e territorialmente vasto como o } \\
\text { Brasil, poder dispor de uma lista de anomalias } \\
\text { prioritárias, selecionadas com base em aspectos } \\
\text { relevantes }\end{array}$ \\
\hline $\begin{array}{l}\text { LIMA, I. D. et } \\
\text { al., } 2017 .\end{array}$ & $\begin{array}{l}\text { Perfil dos óbitos por } \\
\text { anomalias congênitas } \\
\text { no estado do Rio } \\
\text { Grande do Norte no } \\
\text { período de } 2006 \text { a } \\
2013 \text {. }\end{array}$ & $\begin{array}{l}\text { Analisar a prevalência e fatores } \\
\text { associados aos óbitos fetal e } \\
\text { não fetal ou infantil por } \\
\text { Anomalias Congênitas no } \\
\text { Estado do Rio Grande do Norte, } \\
\text { Brasil, no período de } 2006 \text { a } \\
2013 \text {. }\end{array}$ & $\begin{array}{c}\text { Estudo } \\
\text { descritivo. }\end{array}$ & $\begin{array}{l}\text { Os dados apontaram para a maior parte de óbitos por } \\
\text { anomalias congênitas foi do tipo não fetal ou infantil, } \\
\text { sendo a principal anomalia a do sistema } \\
\text { cardiovascular. A maioria dos óbitos ocorreu no } \\
\text { período Pós Neonatal, em crianças com peso entre } \\
2.500 \text { a } 4.000 \text { g. Os óbitos foram prevalentes em } \\
\text { mulheres jovens entre } 20 \text { a } 30 \text { anos, com histórico de } \\
\text { filhos mortos e com idade gestacional entre } 37 \text { a } 41 \\
\text { semanas. }\end{array}$ \\
\hline
\end{tabular}

Fonte: Autores (2021).

Foi possível encontrar que os estudos afirmam uma relação entre a idade da mãe e associação com a ocorrência de anomalias congênitas, sendo a idade é um fator de risco para o desenvolvimento da anomalia congênita. Assim, mães com idade, precoce, existe um nível elevado de possuir malformações do feto devido aos fatores externos com a utilização de medicações para induzir o aborto e o uso do álcool (Avsar et al., 2021).

Em outro estudo, os 1.220 óbitos por anomalias congênitas 67,3\% apresentaram informações para idade materna e idade gestacional, à medida que as doenças infecciosas e deficiência nutrional estão sendo controladas as causas perinatais e as anomalias congênitas são um destaque na saúde pública exigindo medidas específicas para controlar sequelas (Lima et al., 2017).

Estima-se que 15 a 25\% das causas de anomalias congênitas ocorrem devido as alterações genéticas, 8 a $12 \%$ causadas a partir de fatores ambientais e em torno de 20 a $25 \%$ podem ser causadas tanto por alterações genéticas quanto por fatores ambientais, contudo a maioria das anomalias ainda possuem origem incerta (Lima et al., 2017).

De acordo com os estudos, foi examinado associação entre as suplementações de ácido fólico antes e nos primeiros meses de gravidez. Dessa forma, as mulheres que ingeriram suplementos de ácido fólico antes da gravidez e no primeiro mês de gestação possuíam $60 \%$ menos probabilidade de ter recém-nascidos com defeitos do tubo neural (Atlaw et al., 2021). A ingestão alimentar foi encontrada como um fator que aumentam significamente os níveis séricos de folato, sendo uma área amplamente inexplorada (Lee \& Gleeson, 2020). Ou seja, a ingestão do ácido fólico tem se provado um grande contribuinte no combate as más formações, envolvidas no tubo neural.

Com isso a prevalência média de malformações de um caso para cada 100 nascidos vivos, a análise múltipla possui fatores positivos associados a prevalência com o baixo peso ao nascer, gravidez do tipo única, tipo de parto, escore de Apgar insatisfatório e a prematuridade seno relevante para que a prevenção, vigilância e melhorias nos cuidados especializados (Gonçalves et al., 2021).

O tabagismo foi considerado um fator de risco, pois, o consumo de substâncias presentes no cigarro durante a gravidez não possui apenas um impacto a curto prazo, mas, também resultados a longo prazo que podem ser prejudiciais a prole. Nesse estudo, o risco de mortalidade, parto prematuro, defeitos congênitos e a morte perinatal aumentava de acordo com o aumento do consumo de cigarros (Avsar et al., 2021).

Também foi encontrado na presente pesquisa que o risco de Diabetes Mellitus (DM), é conhecido como um fator de risco que pode causar danos ao desenvolvimento fetal e saúde materna. Como encontrado, a malformação congênita é responsável por cerca de aproximadamente 276 mil crianças ao ano em todo o mundo, sendo que 50\% das malformações congênitas sendo atribuídas a fatores nutricionais, genéticos, ambientais e infecciosos. Os fatores ambientais constituem $10 \%$ 
dos casos e com eles os níveis aumentados de glicose na gestação se destacam. Assim, os defeitos congênitos induzidos pelo DM são considerados como significativos problemas de saúde pública (Windt et al., 2021).

O DM é conhecido também como um fator de risco para o aparecimento de distúrbios do tubo neural (DTN), tanto o DM quanto a obesidade são características da síndrome metabólica. Com a presença de algumas características da síndrome metabólica o feto possui um elevado risco em possui DTN. Além disso, as taxas de anomalia congênita em pacientes com diabetes mellitus gestacional aparecem com maior incidência do que na população geral, ocorrendo com maior risco para anomalias específicas como a do tubo neural (Windt et al., 2021).

A gravidade da obesidade materna tem aumentado nos últimos anos, nos países europeus de $7 \%$ a $25 \%$ das mulheres grávidas estão acima do peso, de acordo com os estudos a obesidade materna está associada a resultados adversos na gravidez incluindo assim a malformação congênita, natimorto e prematuro. Com isso, diversos estudos relataram que consistentemente uma relação entre a obesidade materna, sobrepeso e o risco de defeitos congênitos na prole (Helle \& Priest, 2020).

Um estudo constatou que a maior chance de anomalias congênitas se apresenta em gestações múltiplas e, segundo alguns autores, a gemelaridade é um fator importante de defeitos congênitos. O maior número de casos de anomalias congênitas em gestações múltiplas pode ser justificado, em parte, por falhas nas divisões celulares (fatores genéticos) e por fatores ambientais intraútero, constrição de banda amniótica ou de cordão umbilical. Considerando que gestações múltiplas estão associadas ao maior número de partos prematuros (Cosme et al., 2017).

Outro estudo discute sobre o uso do Ibuprofeno profilático para a redução do desenvolvimento da persistência do canal arterial em bebês prematuros, que também é uma das anomalias congênitas, entretanto, possui uma série de efeitos adversos os quais expõe bebês a efeitos colaterais importantes, por isso, não é indicado em forma algum seu uso profilático. Mas, estudos em andamento apontam uma terapia precoce com base em critérios ecocardiográficos nas primeiras 72 horas de vida, que mostram uma alta sensibilidade para o diagnóstico de persistência do canal arterial que provavelmente não fecha espontaneamente. Esses testes estão em andamento em muitas partes do mundo (Ohlsson et al., 2020). São diversas as anomalias que o bebê pode estar exposto, mas, as medidas de tratamento, ainda são estudas em demais casos.

Quando se fala sobre o fechamento cirúrgico da patência do canal arterial (PDA), ainda é considerado na maioria dos casos o tratamento médico devido aos riscos relacionados a cirurgia, nesse estudo 3.779 bebês com muito baixo peso ao nascer, $28 \%$ necessitaram de tratamento para PCA, 75\% foram tratados apenas com indometacina, $8 \%$ apenas com ligadura cirúrgica e $17 \%$ necessitavam de indometacina e ligadura cirúrgica. Assim, bebês com baixo peso ao nascer foram mais propensos a serem tratados cirurgicamente (Ohlsson et al., 2020).

Sendo assim, são evidentes que há diversos fatores os quais podem causar o desenvolvimento de anomalias congênitas, tendo em vista os resultados, o cuidado e atenção ao período do pré-natal é fundamental, para realização de diagnósticos e intervenções diante as anomalias, no entanto, quando a existente após nascimento as principais medidas para que se tenha um cuidado neuroprotetor neonatal é necessário a proteção do sono, parceira com as famílias e um ambiente terapêutico a fim de minimizar o estresse e dor. As intervenções incluem as modificações ambientais como a proteção contra luz, sons excessivos, proteção contra o frio, posicionamento flexionado do RN com a utilização de materiais de suporte evitando assim o manuseio desnecessário e interrupções do sono fornecendo analgesia adequada para procedimentos dolorosos (Peterson \& Evangelista, 2017).

\section{Conclusão}

Dessa forma, é perceptível que as anomalias congênitas estão relacionadas a morbimortalidade infantil, principalmente no período neonatal, tornando assim o seu diagnóstico precoce para o planejamento e alocação de recursos de saúde especializados em pré-natal, natal e pós-natal, para que se tenha a diminuição da morbimortalidade, principalmente neonatal 
precoce, com a finalidade de melhorar a qualidade de vida e dos índices de sobrevida, é necessário que seja disseminado o conhecimento sobre as anomalias e suas causas para que assim muitos casos sejam evitados, apesar da etiologia genética ser um fator contribuinte por uma porcentagem considerável nas anomalias congênitas, deve-se levar em consideração os fatores evitáveis como as infecções que podem ser transmitidas ao feto e endocrinopatias maternas.

Assim, se faz cada vez mais necessário que o tema possa ser conhecido e debatido pela população e profissionais da área da saúde. Pois, os achados encontrados neste estudo, apontam a necessidade de um cuidado especializado e direcionado aos recém-nascidos de modo a evitar a mortalidade, além disso, as consultas com os profissionais que acolhem crianças portadoras de anomalias congênitas, devem cada vez mais instituir um exame físico completo, clínico e sistemático, considerando portanto, uma conduta que deve ser enfatizada e necessária.

\section{Referências}

Aguiar, M. J. B., Campos, A. S., Aguiar, R. A. L. P., Lana, A. M. A., \& Babeto, L. T. (2003). Defeitos do fechamento do tubo neural e fatores associados em recém-nascidos vivos e natimortos. J.Pediatria. 79(2). https://doi.org/10.1590/S0021-75572003000200007.

Alberto, M. V. L., Galdos, A. C. R., Miglino, M. A. \& Santos, J. M. (2010). Anencefalia: Causas de uma mal formação congênita. Revista Neurociência. 18(2), 244-248.

Arruda, T. A. M. Amorim, M. M. R. \&Souza, A. S. R. Mortalidade determinada por anomalias congênitas em Pernambuco, Brasil, de 1993 a 2003. Revista Assoc. Med. Bras. 54(2), 122-6. https://doi.org/10.1590/S0104-42302008000200013.

Atlaw, D., Tekalegn, Y., Sahiledengle, B., Seyoum, K., Solomon, D., Gezahegn, H., Tariku, Z., Tekle, Y., \& Chattu, V. K. (2021). Magnitude and determinants of neural tube defect in Africa: a systematic review and meta-analysis. BMC Pregnancy and Childbirth. 21(426). https://doi.org/10.1186/s12884-021-03848-9.

Andrade, A.M., Ramalho, A.A., Opitz, S.P., Martins, F.A \& Koifiman, R.J. (2017). Anomalias congênitas em nascidos vivos. Revista Brasileira em Promoção da Saúde. vol. 30. https://doi.org/10.5020/18061230.2017.6309.

Avsar, T. S., McLeod, H. \& Jackson, L. (2021). Health outcomes of smoking during pregnancy and the postpartum period: an umbrella review. BMC Pregnancy and Childbirth. 21(254). https://doi.org/10.1186/s12884-021-03729-1.

Brasil, Ministério da Saúde. Anomalias congênitas no Brasil, 2010 a 2019: análise de um grupo prioritário para a vigilância ao nascimento. Boletim epidemiológico, 52(6), p. 1-22.

Cosme, H. W., Lima, L. S. \&Barbosa, L. G. (2017). Prevalência de anomalias congênitas e fatores associados em recém-nascidos do município de São Paulo no período de 2010 a 2014. Revista Paul. Pediatria. 35(1). https://doi.org/10.1590/1984-0462/;2017;35;1;00002.

Garcia, A. G. P. (1981). Causas infecciosas das anomalias congênitas. Inst. Oswaldo Cruz. 76(3), 307-328. https://doi.org/10.1590/S0074-02761981000300010.

Gonçalves, M. K. S., Cardoso, M. D., Lima, R. A. F., Oliveira, C. M. \& Bonfim, C. V. (2021). Prevalência e fatores associado sãs malformações congênitas em nascidos vivos. Acta Paul. Enfermagem. 34. https://doi.org/10.37689/acta-ape/2021AO00852.

Helle, E. \& Priest, J. R. (2020). Maternal obesity and diabetes mellitus as risk factors for congenital heart disease in offspring. J Am Heart Assoc. 9(8). 10.1161/JAHA.119.011541.

Lee, S. \& Gleeson, J. G. (2020). Closing in on mechanismof open neural tube defects. Trends in Neurosciences. 43(7), p. 519-532. 10.1016/j.tins.2020.04.009.

Lima, I. D., Araújo, A. A., Medeiros, W. M. C., Feitosa, J. M., Silva, R. B., Maia, E. F. T., \& Wingerter, D. G. (2017). Perfil dos óbitos por anomalias congênitas no estado do Rio Grande do Norte no período de 2006 a 2013. Rev. Ciên. Méd. Biológicas. 16(1), 52-58. http://dx.doi.org/10.9771/cmbio.v16i1.17422.

Lima, L. M. M., Vianna, R. P. T. \& Moraes, R. M. (2019). Identificação das anomalias congênitas baseado em um modelo de decisão a partir de redes neurais artificiais. J. Health Inform. 11(1), p. 8-12.

Ohlsson, A., Walia, R. \&Shah, S. (2020). Ibuprofeno para tratamento de persistência do canal arterial em recém-nascidos prematuros e/ou de baixo peso. Cochrane Database of Systematic Reviews. 2. 10.1002/14651858.CD003481.pub8.

Peterson, J. K. \& Evangelista, L. S. Developmentally supportive care in congenital heart disease: A Concept Analysis. J Pediatr Nurs. 36, $241-247$. 10.1016/j.pedn.2017.05.007.

Pawluk, M. S., Campaña, H., Gili, J. A., Comas, B., Giménez, L. G., Villalba, M. I., Scala, S. C., Poletta, F. A., \& López Camelo, J. S. (2014). Determinantes socials adversos y riesgo para anomalias congênitas seleccionadas. Arch Argent Pediatr. http://dx.doi.org/10.5546/aap.2014.215.

Rocha, G., Pinto, S., Pinto, J.C., Monteiro, J., Guedes, M.B \& Guimarães, H. (2004). Anomalias congénitas gastrintestinais e da parede abdominal. Revista do Hospital de criança Maria Pi.v13. https://repositorio.chporto.pt/bitstream/10400.16/614/1/Anomalias\%20cong\%c3\%a9nitas\%20gastrintestinais.pdf.

Santos, A. C. C. \& França, G. V. A. Lista de anomalias congênitas prioritárias para a vigilância no âmbito do Sistema de Informações sobre nascidos vivos no Brasil. (2021). Epidemiol. Serv. Saúde. 30(1). https://doi.org/10.1590/S1679-49742021000100030.

Souza, M. T., Silva, M. D. \& Carvalho, R. Revisão integrativa: o que é e como fazer? Einstein. 8, 102-6. https://doi.org/10.1590/S1679-45082010RW1134.

Windt, M. V. D., Schoenmakers, S., Van Rijn, B., Galjaard, S., Steegers-Theunissen, R., \& Van Rossem, L. (2021). Epidemiology and (Patho) physiology of folic acid supplement use in obese women before and during pregnancy. Nutrients. 13(2), 331. 10.3390/nu13020331. 